\title{
Explotación, valor, conocimiento y trabajo en la producción y reproducción de contenidos educativos en línea ${ }^{1}$
}

Mariano Zukerfeld ${ }^{2}$

Recibido: 30/08/2021; Aceptado: 18/10/2021

Cómo citar: Zukerfeld, M. (2021) Explotación, valor, conocimiento y trabajo en la producción y reproducción de contenidos educativos en línea. Revista Hipertextos, 9(16), 55-76. https://doi.org/10.24215/23143924e040

Resumen. Este artículo intenta abordar la explotación por reproducción de trabajadores docentes que preparan contenidos para educación en línea en procesos productivos con fines de lucro. El enfoque teórico se basa en una definición y una tipología de diferentes tipos de explotación. La evidencia empírica proviene de dos encuestas cortas gemelas respondidas por 129 académicos con sede en el Reino Unido y Argentina. Concluimos que los docentes tienden a asociar la remuneración esperada con el tiempo dedicado a preparar las clases -más que con sus conocimientos-, lo que ofrece una base favorable al florecimiento de la explotación por reproducción.

Palabras Clave: explotación, conocimiento, valor, trabajo, educación

Sumario. 1. Introducción: Educación online y trabajo docente en la pandemia. 2. Marco teórico: antecedentes, definición y tipos de explotación. 3. Metodología. 4. Resultados de la encuesta: tiempo, conocimiento, remuneración y titularidad de los contenidos de la educación en línea. 5. Conclusiones.

Exploitation value, knowledge and labour en the production and reproduction of online educational content

Abstract. This article intends to tackle, the capitalist exploitation through reproduction of teachers producing content for online courses in for profit productive processes. The theoretical approach relies on a definition and a tipology of different kinds of exploitation. The empirical evidence comes from two twin

${ }^{1}$ El trabajo de campo de esta investigación fue financiado por el WIAS de la University of Westminster. Vaya mi más profundo agradecimiento para esta institución y su director, Christian Fuchs que brindaron el apoyo económico, el espacio físico, los ámbitos de intercambio y a su vez importantes sugerencias para fortalecer esta investigación aun cuando los resultados difirieran de sus propias opiniones teóricas. Agradezco también a los dos revisores anónimos por sus aportes a este artículo.

2 Investigador del CONICET (Argentina) y del equipo e-TCS del Centro CTS, Umai. Profesor Adjunto de la Carrera de Sociología (UBA), de la Maestría en Propiedad Intelectual de FLACSO Argentina, de la Maestría en Ciencia Tecnología y Sociedad de la UNQ. Contacto: marianozukerfeld@gmail.com 
short surveys answered by 129 academics based in the UK and Argentina. We conclude that teachers tend to associate their expected remuneration with time spent preparing classes -rather than their knowledge-, which enables exploitation through reproduction.

Keywords: exploitation, knowledge, value, labour, education

\section{Exploração, valor, conhecimento e trabalho na produção e reprodução de conteúdos educacionais online}

Resumo. Este artigo tenta abordar a exploração por reprodução de docentes que elaboram conteúdos para a educação online em processos produtivos com fins lucrativos. A abordagem teórica baseia-se na definição e tipologia dos diferentes tipos de exploração. A evidência empírica vem de duas pesquisas curtas gêmeas respondidas por 129 acadêmicos baseados no Reino Unido e na Argentina. Concluímos que os professores pretendem associar a remuneração esperada com o tempo despendido na preparação das aulas - e não com os seus conhecimentos -, o que oferece uma base favorável para o florescimento da exploração pela reprodução.

Palavras-chave: exploração, conhecimento, valor, trabalho, educação

\section{Introducción: Educación online y trabajo docente en la pandemia}

La pandemia de COVID 19 indudablemente ha profundizado y radicalizado tendencias hacia la digitalización y mercantilización de esferas de actividad que, ciertamente, la precedieron por mucho. En otros términos, ha acelerado el ritmo de la subsunción de la vida social al capitalismo informacional. La educación superior es una de las esferas en las que tales tendencias se han vuelto tan obvias como masivas durante 2020 y 2021, y sobre la que, pasada la pandemia, habrán dejado efectos indelebles (Izumi et al, 2021; Zhou et al, 2020; Mishra et al, 2020; Castillo, 2020; Marotias, 2020; Gagliardi, 2020). Sin embargo, ya bastante antes de la pandemia la educación a distancia, particularmente a través de plataformas online había sido un tema ampliamente estudiado en el contexto del capitalismo informacional, tanto a nivel mundial (Harasim, 1996; Weller, 2002; Anderson, 2008; Salmon, 2013; Browne, Jenkins and Walker, 2006) como en América Latina (por ejemplo la compilación de Torres y Rama, 2010). Generalmente, se discutía la masificación y el abaratamiento del aprendizaje, las posibilidades de inclusión social que brinda, pero también las dificultades para su implementación efectiva. En el plano de la educación informal, ya se había indagado respecto del aprendizaje a través de videos tutoriales, foros, juegos (Lee y Lehto, 2013; Selwyn, 2007). En cambio, en la educación formal y no formal, el aprendizaje online tomaba la forma, típicamente, de los MOOC (Massive Open Online Course) o los VLE (Virtual Learning Enviroment) (Cooper y Sahami, 2013; Hoxby, 2014; Borrego et al, 2008). La masificación de estas formas de educación online resultaba evidente desde la segunda década del nuevo milenio. En el caso de la educación formal superior, por ejemplo, en los EE.UU. había en 2013 un 27\% que tomaban alguno o todos sus cursos a distancia. Particularmente, en el caso de la educación privada con fines de lucro, ese porcentaje alcanzaba al 59,3\% en 2013 (US Department of Education, 2016). A nivel mundial, en ese año, los proveedores de educación privada online formal y no formal obtuvieron ingresos por 56.200 millones de dólares (Santamans, 2014). 
Sin embargo, en este área de la educación formal superior con fines de lucro, tanto antes como durante la pandemia se ha estudiado escasamente la explotación de los docentes, particularmente de los que producen los contenidos de las clases. En términos teóricos, la explotación tiene lugar tanto en la educación formal (en la que los profesores universitarios son invitados o forzados a dictar cursos online) como en la no formal, que involucra a docentes contratados por consultoras que producen cursos ad hoc, típica pero no exclusivamente para las demandas de sus empresas clientes).

En efecto, desde una perspectiva materialista, en ambos casos sucede lo mismo: el capital busca la traducción de los saberes desde un soporte subjetivo (la individualidad del profesor o del capacitador) hacia una codificación como bien informacional (los textos, videos, software, etc.). Esta traducción tiene consecuencias económicas y jurídicas notables. Por un lado, lo que antes se proveía estrictamente como un servicio y que por ende debía ser pagado en cada ciclo lectivo, ahora se ha vuelto un bien; por cierto, un bien muy particular, que puede reproducirse con costos cercanos a 0 , pudiendo replicarse sin tener que remunerar nuevamente al docente que escribiera el curso. Esto surge del hecho jurídico fundamental: la titularidad de los contenidos deja de ser del docente, y pasa a ser de la empresa. Tal cesión de derechos se encuadra dentro de legislaciones de derechos de autor o, más frecuentemente, de contratos específicos ${ }^{3}$.

Aunque tanto en la modalidad presencial como en la online los docentes que crean contenidos reciben una compensación económica, en ambos casos estamos usualmente frente a situaciones de explotación: el valor que reciben esos docentes es menor que el que producen. Sin embargo, aquí nos interesa llamar la atención respecto de que en cada caso predomina un tipo de explotación distinta: en la modalidad presencial, se ejerce la explotación por enajenación (basada en la apropiación impaga de unidades de tiempo de trabajo; en cambio, en la modalidad online, toma forma la explotación por reproducción (basada en la codificación impaga de conocimientos y la titularidad directa de esos conocimientos por parte de la empresa, ver marco teórico).

Conceptualizar a la educación virtual mediante la noción de explotación por reproducción resulta extremadamente importante políticamente porque los docentes (y las instituciones que los nuclean) por lo general no perciben con claridad el carácter de las relaciones sociales en las que están inmersos ni cómo lidiar jurídicamente con ellas. Naturalmente, toda vez que durante la pandemia la producción de contenidos para educación a distancia o, más precisamente, educación virtual de emergencia, se ha masificado de modo notable, estas cuestiones se han vuelto más acuciantes.

La limitación en el diagnóstico económico y político de los docentes podría surgir de un problema teórico tácito: están (y estamos) acostumbrados a asociar el valor (y en última instancia sus ingresos, sus salarios, etc.) al tiempo de trabajo. Sin embargo, en la producción de bienes informacionales resulta evidente que la ganancia del capitalista no depende única ni principalmente del tiempo de trabajo de los docentes.

De este modo, en entrevistas exploratorias habíamos encontrado reiteradamente situaciones en las que las empresas educativas pagaban a los docentes por desarrollar los contenidos más, en términos de remuneración por hora, de lo que estos cobraban por cada hora de trabajo

\footnotetext{
3 Como lo explica un portal destinado a "ayudar" a los docentes online: "Most online colleges require course developers to sign a "work-for-hire" agreement which gives the college the right to own the course. This means you cannot freely take the course and use it to teach elsewhere. The course will belong to the college, not you" (Geteducated.com, 2016).
} 
presencial (considerando en ambos casos todos los tiempos involucrados). Los docentes, que evaluaban su situación en términos de la explotación por enajenación, esto es, en términos de cuánto reciben por hora de trabajo, percibían la situación como satisfactoria. $\mathrm{O}$ al menos, como menos insatisfactoria que las clases presenciales. Sin embargo, usualmente los docentes no consideraban que su curso sería usado en reiteradas ocasiones y que, probablemente, el lucro obtenido por los empleadores podría ser mucho más elevado. Así, de manera más general, los docentes y los sindicatos que los representan, aferrados explícita o implícitamente a la relación entre tiempo y valor, dejan de percibir que sus ingresos no surgen de un servicio, sino de la codificación y reproducción de sus saberes en determinados soportes.

Partiendo de ese diagnóstico, en 2017, con el apoyo del Westminster Institute for Advanced Studies y bajo la dirección de Christian Fuchs, realicé una pequeña encuesta sobre la forma en que los docentes de educación superior que se desempeñan principalmente en Argentina e Inglaterra se representan las remuneraciones que reciben por los contenidos que producen para la educación online. Más específicamente, quise analizar la hipótesis que sugería la evidencia anecdótica previa y el marco teórico en el que me baso: que los docentes tienden a asociar el valor mucho más con el tiempo de trabajo que con los conocimientos involucrados. De confirmarse esta hipótesis, se trataría de una importante base ideológica para favorecer los modelos de explotación por reproducción, en boga en el capitalismo informacional y, posiblemente, amplificados durante y luego de la pandemia de Covid 19.

Los resultados preliminares de la encuesta se expresaron en un extenso informe de investigación que, sin embargo, no se ha traducido en publicaciones en revistas académicas en inglés ni español (Zukerfeld, 2017a) en parte por motivos contractuales. Es por eso que este artículo presenta por primera vez parte de esos resultados empíricos en formato de artículo, entendiendo que no sólo conservan vigencia, sino que podrían resultar oportunos para avanzar en la agenda teórica y política de la pospandemia.

Así, este trabajo busca operacionalizar y contrastar reflexiones teóricas con resultados empíricos. Algunas de las preguntas a las que este trabajo intenta responder son: ¿cómo se relaciona el pasaje de algunas actividades docentes desde servicios hacia bienes informacionales con diferentes formas de explotación capitalista? ¿Qué conceptos de una teoría de la explotación pueden dar cuenta de ambas situaciones? ¿Basta con una teoría del valor que asocie valor a tiempos para comprender esas distintas formas de explotación? ¿Podría, de hecho, funcionar esa asociación entre valor y tiempo de trabajo como un mecanismo ideológico que favorece ciertas formas de explotación, particularmente de los docentes que producen contenidos para la educación online? Así, la pregunta crucial es: ¿cómo se representan los docentes el origen del valor económico de los contenidos que producen para la educación online? ¿Lo relacionan con el tiempo de trabajo o con los conocimientos involucrados? ¿Qué tanto tienen en cuentan la reproducción de los contenidos y el lucro que pueden generar? ¿Qué representaciones tienen sobre la titularidad de esos contenidos? ¿En qué medida distinguen la titularidad de los contenidos dictados oralmente en clases presenciales de la de los contenidos de las clases a distancia?

El resto del presente artículo está organizado del siguiente modo: la segunda sección presenta una breve discusión teórica sobre el sentido que se les da aquí al concepto de explotación y otros conexos. La tercera sección introduce consideraciones metodológicas sobre la encuesta realizada mientras que la sección cuarta analiza la información resultante de la misma. Finalmente, en la quinta sección se presentan las conclusiones. 


\section{Marco teórico: antecedentes, definición y tipos de explotación}

Nuestra propuesta en relación a la explotación se basa en una perspectiva materialista cognitiva ${ }^{4} \mathrm{y}$, en particular, en una teoría del valor-conocimiento ${ }^{5}$.

Respecto de la explotación que ocurre en el capitalismo informacional en general y específicamente sobre docentes que producen contenidos para educación online, recuperamos críticamente varios antecedentes. En primer lugar, la noción de explotación capitalista de Marx ([1867] 1990) y el marxismo (Hilferding, [1910] 1981; Sweezy, 1942), del que mantenemos la necesariedad, en todas las etapas del capitalismo, de la explotación, entendida como una relación de intercambio legal y libre, de magnitudes objetivamente asimétricas. Por otro lado, los abordajes Sraffianos (Hodgson, 1988; Garegnani, 1979) y del marxismo analítico (Roemer, 1985; Elster, 1981; Wright, 1985). De éstos retomamos el énfasis en una teoría de la explotación que no se base necesariamente en la teoría del valor trabajo de Marx y la vocación sistemática del análisis. Asimismo, los autores autonomistas y del capitalismo cognitivo han rozado el tema (Fumagalli, 2015; pero especialmente, Moulier-Boutang, 2011). En tercer lugar, desde el materialismo cultural y perspectivas afines (Fuchs, 2010, 2012, 2015; Fisher, 2012) recuperamos por un lado la idea de que las plataformas capitalistas de Internet funcionan en base a la explotación capitalista de sus usuarios (aunque no sólo de ellos) y, por otro, la de actualizar el aporte de Smythe (1977) para concebir un tipo de explotación de las audiencias. Finalmente, en términos de antecedentes locales, este trabajo continua investigaciones que previamente intentaron avanzar sobre la dimensión cognitiva de la explotación (Kreimer y Zukerfeld, 2014; Zukerfeld, 2014; Rabosto, 2014; Yansen, 2015; Dolcemáscolo, 2014; Dolcemáscolo y Yansen, 2017; Liaudat, 2021)

\footnotetext{
${ }^{4}$ El materialismo cognitivo sostiene la asunción básica de las filosofías materialistas: todos los entes reales son materiales, y sigue en particular los emergentistas, pero se distingue por afirmar que los bienes y sujetos están hechos de combinaciones variables de materia/energía y conocimientos. Así, el conocimiento sólo existe en un soporte de materia/energía. El soporte material del conocimiento le confiere propiedades onotológicas, económicas y jurídicas diferenciales a cada forma concretamente existente de conocimiento. Por ejemplo, que la idea de "rueda" exista subjetivamente como representación mental individual, como objetivación en un artefacto determinado o como codificación en un texto, confiere a ese conocimiento posibilidades muy disímiles de, por caso, difundirse, ser considerado eficaz o caer en el olvido. El materialismo cognitivo analiza flujos y stocks de distintos tipos de conocimeintos (en base a sus soportes materiales) y materia/energía, especialmente respecto de las regulaciones que pesan sobre ambos: propiedad intelectual y propiedad física, respectivamente. Para un desarrollo mayor véase Zukerfeld, 2010, 2017b.

${ }^{5}$ La idea de una teoría del valor conocimiento fue sugerida por autores como Bell y Jaros, se ubica dentro de las teorías objetivas del valor, y sostiene que el único ente que crea riqueza es el conocimiento (en sus variadas formas), mientras que la materia/energía solo se transforma. Esto implica dos divergencias respecto de la teoría del valor trabajo de Marx. Por un lado, el trabajo (como señalara con claridad Bentham) está compuesto de energía y conocimientos. Así, en nuestra perspectiva, no es el trabajo en general el que crea más valores que los que consume, sino que es sólo su componente cognitivo el que presenta esa propiedad ontológica que permite la existencia eventual del plusvalor. El trabajador recibe como salario los ingresos que le permiten reponer las energías consumidas (el "valor de cambio" de la fuerza de trabajo) mientras que el hecho de que sus destrezas no se desgasten con su uso es el que permite que el capitalista se apropie de esos frutos sin pagar por ellos más que parcialmente. El valor de uso de la fuerza de trabajo incluye la energía y el conocimiento, pero el capitalista sólo paga por el primero. Así, la plusvalía en el sentido marxiano puede comprenderse como el conocimiento que se objetiva en el producto de la actividad laboral. Por otro lado, existe creación de valor por parte de conocimientos que no son necesariamente reducibles a unidades de trabajo. Los flujos cognitivos que crean valor ocurren parcial pero masivamente en la presente etapa del capitalismo fuera del tiempo de trabajo. La distinción entre estos aspectos es fundamental para superar dificultades que la teoría del valor trabajo encuentra en el capitalismo informacional (como el valor que añade el software, el caracter productivo del trabajo de cuidados, etc.).
} 
En este contexto, definimos sintéticamente a la explotación capitalista y sus tres modalidades.

La explotación puede definirse, simplificadamente, como una relación de intercambio asimétrico de recursos entre dos tipos de actores, Explotadores (E) y explotados (e), que ocurre al interior de procesos productivos y que resulta en que los actores $\mathrm{E}$ obtienen una ventaja económica, un plusvalor objetivo a expensas de los explotados, independientemente de las representaciones subjetivas que ambos tipos de actores tengan respecto de las relaciones en las que participan. En la explotación capitalista en particular, se añaden dos rasgos: Por un lado, los procesos productivos en cuestión están orientados a la producción de mercancías; específicamente, los actores E actúan en esos procesos (incluyendo a la relación con los actores e) con el fin principal -sino único- de obtener un lucro. Por otro, esas relaciones ocurren en mayor o menor medida, de modo consentido y son no-ilegales: no implican la violación clara, evidente e indisputable de ninguna legislación vigente.

La explotación capitalista adopta tres modalidades:

Explotación por Enajenación: Determinados conocimientos de los actores e son objetivados durante el tiempo de trabajo en un producto que es enajenado por el actor E. Este es el concepto tradicional de explotación, con dos salvedades: la clave está en los conocimientos objetivados en el producto (que son la fuente de plusvalor) y que esta modalidad incluye no sólo a lo que ocurre al interior de la unidad productiva sino también a los productos de trabajadores tercerizados o autónomos.

Explotación por Reproducción: Determinados conocimientos de los actores e son copiados por el actor E, que se vuelve titular de esos saberes mediante marcos normativos o contractuales. Esto sucede típicamente cuando el capital se apropia (a veces mediante la codificación) de conocimientos sin un pago específico que compense a los explotados por la reproducción, obras derivadas y otros usos posteriores de esos conocimientos.

Explotación por Atención: Determinados conocimientos vehiculizados por los actores E son subjetivados hacia los actores e. Aquí se trata del camino inverso al de las otras dos modalidades: especialmente, en una economía en la que el recurso escaso es la atención humana (Simon, 1996), se aprovecha sin compensación suficiente la atención de las audiencias (Smythe, 1977; Fuchs, 2010) que se vende a las empresas de publicidad (usualmente, en combinación con los datos obtenidos mediante la explotación por reproducción), para inyectarle flujos cognitivos particularizados. Es fundamental señalar que las tres modalidades no son mutuamente excluyentes, sino que actúan en muchos procesos productivos de manera combinada.

Como se indica en la introducción, en este trabajo el foco está en la explotación por reproducción, por lo que conviene profundizar en esta categoría.

Lo que aquí se define como explotación por reproducción surge de haber detectado repetidamente anomalías en el concepto tradicional de explotación (que sólo comprende lo que aquí llamamos explotación por enajenación). En efecto, en la historia de las empresas, ramas industriales y países que han tenido éxito en la acumulación de capital, hay formas que no pueden reducirse a la explotación por enajenación -en las que el capital depende críticamente de la mediación del producto y del tiempo de trabajo). Los procesos de acumulación de estas empresas y países se basan fundamentalmente en la copia no remunerada de conocimientos de las más diversas fuentes, como han documentado varios estudios (Chang 2001; Drahos y Braithwaite 2002; May and Sell 2006; Johns 2010). Se trata de conocimientos que se codifican en textos, se objetivan en máquinas e incluso se alojan en subjetividades reguladas por contratos específicos, en todos los casos bajo la propiedad del capital. 
Mientras que la explotación por enajenación requiere la relación entre el explotado y el producto de su trabajo como medio para acceder a los conocimientos que posee el primero, en la explotación por reproducción la captura de este conocimiento se vuelve independiente de esta relación. Por esta razón, es tentador involucrarse en el -quizás extenuado- ejercicio de la paráfrasis marxiana y afirmar que la explotación por enajenación representa la subsunción formal del conocimiento bajo el capital, mientras que la explotación por reproducción nos presenta la subsunción real del conocimiento bajo el capital.

Es importante subrayar que la explotación por reproducción puede tener lugar dentro o fuera del proceso laboral, y los conocimientos implicados pueden surgir de contextos bastante heterogéneos: conocimientos subjetivos de los científicos, conocimientos tradicionales o populares, conocimientos codificados como información digital y, por supuesto, conocimientos asociados a las habilidades laborales.

La idea de que los trabajadores poseen conocimientos que no se objetivan en el producto del trabajo pero que son igualmente cosechados por las empresas sin una compensación adecuada se ha considerado al menos desde Marx, desde dos ángulos. El primero está relacionado con la organización del proceso de trabajo. Por ejemplo, Coriat (1985), en su análisis del taylorismo, ha demostrado cómo romper el monopolio de los conocimientos de los trabajadores fue una tarea decisiva para el establecimiento del capitalismo industrial norteamericano. El segundo enfoque se basa en la noción de "intelecto general" mencionado por Marx en los Grundrisse. Este es el curso tomado por el autonomismo italiano (Lazzaratto y Negri, 2001) y la teoría del capitalismo cognitivo (Vercellone, 2011; Moulier-Boutang 2011). En la etapa actual del capitalismo, se afirma, los trabajadores producen conocimientos valorizables a lo largo de su vida y que las empresas se apropian de ellos a través de la relación laboral. Por supuesto, en general, esta literatura da por sentada la propiedad corporativa del conocimiento y no cuestiona si las compensaciones a los trabajadores son apropiadas o no.

Más allá de la regulación, la clave de la explotación a través de la reproducción es siempre la traducción de los saberes de un soporte a otro. Mientras los conocimientos son transportados por la subjetividad de un trabajador, la empresa tiene un control muy limitado: solo puede acceder a ellos mediante la relación laboral contingente con el trabajador que los acarrea. Por tanto, la traducción hacia soportes que faciliten la propiedad de la empresa es imperativa respecto de los conocimientos críticos. La traducción más significativa es la que implica codificación, es decir, traducción de conocimientos desde la subjetividad de la mente individual a diversos tipos de información (cuyos derechos de autor pertenecerán a la empresa): textos, videos, etc.

Estas cuestiones afectan especialmente a los trabajadores que a veces se etiquetan como "cognitivos". Imaginemos a un músico profesional, por ejemplo una pianista, contratada por un productor. Cuando presta un servicio (acompañando a un cantante en una actuación en vivo), por el que percibe una remuneración, si todo se desarrolla adecuadamente será explotada por enajenación: su tiempo de trabajo será el vehículo que lleve sus conocimientos valiosos por los cuales el productor o su empresa obtienen una plusvalía. Para volver a explotarla (a la pianista), sus servicios serán necesarios espectáculo tras espectáculo. Pero cuando esta música es contratada para grabar, es decir, para que sus conocimientos subjetivos se traduzcan en codificación como información digital, la situación se vuelve bastante diferente, y toma la forma de explotación por reproducción: la empresa puede copiar y vender esos conocimientos objetivados tantas veces como sea quiera, con costos marginales tendientes a cero, y usualmente sin ninguna compensación adicional otorgada a la parte explotada. Esto permite que el pago por 
una grabación sea mucho más alto que el pagado por una actuación en vivo y manteniendo una tasa de explotación similar o aun mayor. Sin embargo, para los trabajadores, sindicatos y académicos que observan la situación con los lentes de la explotación por enajenación (pago por hora), parecería que no hay explotación.

La explotación por reproducción está expandiéndose en el capitalismo informacional. La podemos encontrar no solo en el mundo del arte y el entretenimiento sino también en la producción de software y en lo que nos ocupa en este artículo: la educación formal y no formal. El proceso de transformación de la educación comercial tradicional presencial (que como señalamos más arriba implica la explotación por enajenación) hacia la educación virtual permite una analogía simple con el caso de la performance en vivo y la grabación en la música. En efecto, simplificando un poco las cosas y concentrándose en el ejemplo de un docente que es filmado o que escribe contenidos, se les paga una sola vez (al igual que el músico de sesión) por "clases virtuales" que se reproducen repetidamente. La empresa educativa paga al docente por su "tiempo de trabajo" algo más de lo que recibiría por una clase presencial, pero incrementará la cantidad de estudiantes que pagarán por ese contenido en una proporción sustancialmente mayor. El docente productor de contenidos, naturalmente, podría percibir que se trata de un cambio excelente, hasta que quede desempleado, subempleado, precarizado, etc.

De este modo, la medición del valor (y la expectativa de remuneración) en términos de (cualquier tipo de) tiempo no es suficiente para evitar la explotación por reproducción. De hecho, una remuneración adecuada en términos de tiempos, es decir, que no implica explotación por enajenación, puede permitir (e incluso legitimar) la explotación de los conocimientos copiados, esto es, la explotación por reproducción.

Para que la explotación por reproducción funcione sin fricciones, el capitalismo necesita la ayuda de algunas creencias ideológicas. En el caso de la producción de contenidos digitales, es decir, de bienes informacionales que se reproducen con costos cercanos a 0 , relacionar la remuneración -y quizás en última instancia el valor- con el tiempo podría encajar en esta necesidad ideológica.

Esto conlleva, claro está, una paradoja o mejor, una ironía histórica. La asociación entre tiempo y trabajo, si bien fue alumbrada por David Ricardo, está ampliamente identificada con Marx, con su análisis crítico del capitalismo y, particularmente, con su versión de la teoría del valor trabajo. Así, una idea que funcionó como base para sustentar y organizar luchas de los trabajadores del capitalismo industrial contra su explotación podría estar, inversamente, funcionando como herramienta ideológica que permite explotarlos durante el capitalismo informacional. No se trata de otra cosa que de la historicidad de las categorías teóricas, otra de las contribuciones fundamentales a las ciencias sociales de Marx a la que, en otra ironía notable, muchos marxistas resultan más bien impermeables.

\section{Metodología}

Las principales fuentes primarias de esta investigación fueron dos encuestas cortas gemelas, en español e inglés, realizadas en línea en abril y mayo de 2017. Se trató de una muestra autoseleccionada de 129 docentes, profesores e investigadores de educación superior a los que se llegó mediante una invitación por correo electrónico para completar la encuesta en base a listas previamente identificadas. Los encuestados en español (37) eran principalmente de Argentina, pero algunos de ellos se encontraban en otros países de América Latina. Del mismo modo, los 
encuestados en inglés (92) tenían mayormente su base en el Reino Unido, pero varios de ellos se hallaban en otros países europeos y los EE. UU. En términos de sexo, edad y disciplinas académicas ambas muestras eran similares y comparables. No obstante, el número total de respuestas es bajo y su representatividad desconocida, por lo que no se pueden realizar generalizaciones a ninguna población y los resultados deben tomarse con cautela. Están destinados a sugerir hipótesis, no a corroborarlas.

¿Por qué realizar encuestas en la Argentina y el Reino Unido? Resumidamente, por un lado, por los fuertes contraste entre un sistema educativo de un país periférico y el de uno central, es decir, para ver en qué medida las diferencias entre ambos se expresan en las representaciones de los y las docentes sobre el origen del valor de los contenidos que producen. Por otro lado, por las coincidencias: en ambos casos se observaba ya en 2017 el ascenso vertiginoso de la educación online. Esto se expresaba, por ejemplo, en las universidades que lideraban la matrícula total y/o los nuevos inscriptos, que básicamente presentan modelos dominados por la educación a distancia. En Argentina, la Universidad Siglo 21 ya era la que mayor cantidad de estudiantes presentaba de entre las privadas y mostraba un mayor crecimiento de todo el sistema. En el Reino Unido, la Open University era la que contaba con mayor cantidad de estudiantes de todo el sistema. Asimismo, en ambos casos el lucro está presente. El hecho de que las universidades se presenten formalmente en muchos casos como organizaciones sin fines de lucro (como charities en todos los casos en el Reino Unido) no obsta para que produzcan mercancías educacionales y estén animadas por la búsqueda de ganancias.

El cuestionario estaba compuesto por 11 preguntas estructuradas y un espacio abierto para comentarios adicionales. Fue ideado para ser llenado en un tiempo promedio de 5 minutos. Este fue un requisito ex ante importante para intentar disminuir el sesgo hacia el grupo particular de encuestados que están dispuestos a dedicar una cantidad considerable de tiempo a responderla. Se pidió a los encuestados que expresaran sus primeros pensamientos, lo que es útil para captar representaciones intersubjetivas inmediatas e incluso inconscientes que dan forma a las ideologías.

En cuanto a las preguntas específicas, varias de ellas se relacionan con el objetivo señalado: auscultar las representaciones respecto del origen del valor económico de los contenidos educativos producidos. La pregunta principal en este sentido era: "Supongamos que te ofrecen preparar los contenidos de un curso virtual para una institución educativa que cobrará a sus alumnos por el acceso a este curso. ¿Cuál es la principal variable de la que depende la magnitud del pago que aceptarías por esa tarea?’. La principal tensión que estructura esta pregunta y las categorías de respuestas a ella que utilizamos, es la que existe entre el tiempo y el conocimiento como fuentes últimas del valor. Siendo la pregunta principal, presentamos la operacionalización realizada. Naturalmente, existen varios tipos de tiempo y conocimientos relacionados con las remuneraciones en la educación en línea. Proporcionamos a los encuestados dos opciones relacionadas con el tiempo. Por un lado, "El tiempo dedicado a la preparación de los contenidos para ese curso", por otro, "El tiempo de estudio que tuve sobre el tema y/o mi experiencia como docente". En cuanto al conocimiento, y con base en investigaciones previas, operacionalizamos cuatro tipos ${ }^{6}$. El primero es "Los conocimientos que tengo sobre el tema y/o mi prestigio como docente". Esto incluye el conocimiento subjetivo y el reconocimiento (un tipo de conocimiento intersubjetivo), en particular, alude al ser recompensado por el grado de atención conquistado y

\footnotetext{
${ }^{6}$ Respecto de los distintos tipos de conocimientos aludidos en lo que sigue, vease Zukerfeld, $2017 \mathrm{~b}$.
} 
el capital social o las redes construidas. La segunda opción era, de alguna manera, la opuesta, en el sentido de que no se refería al conocimiento que ya poseen los académicos, sino al conocimiento (reconocimiento intersubjetivo) que podrían recibir, como compensación (parcial) por sus cursos: "El prestigio, las conexiones y/o la experiencia que me da esa actividad docente". Las otras dos opciones se relacionan con el hecho de que el conocimiento que brindan los académicos se objetiva materialmente como un bien informacional, es decir, que los contenidos del curso pueden ser copiados y comercializados de manera reiterada. Ambas apuntan, de distinto modo, a los ingresos que podría obtener la institución educativa al aprovechar esos conocimientos digitalizados. La quinta opción dice: "La cantidad de alumnos que van a recibir el curso y/o la magnitud de dinero que recauda la institución que te contrata"

Esto refiere, entonces, a las ganancias y solo indirectamente al hecho de que el curso podría reproducirse. De hecho, esta opción es amplia y también podría estar relacionada con las clases presenciales. La sexta y última opción era más específica: "La cantidad de veces que se va a utilizar el curso", y se refería inequívocamente a la replicabilidad de conocimientos objetivados como bienes informacionales.

Complementariamente, la encuesta incluía una pregunta acerca de las razones que explicaban la elección de las respuestas a la pregunta previa, al efecto de controlar y profundizar en las mismas. Además de razones relacionadas con las categorías utilizadas en la pregunta anterior, se incluyó una categoría relativa a la incidencia de terceros ("porque es lo que consideran otros colegas y/o el sindicato") y una "Otros" que permitía completar un campo libre

Por otro lado, incluimos en el presente trabajo también las respuestas a una pregunta sobre la titularidad/propiedad de los contenidos preparados por los docentes. Nos interesaba conocer las representaciones sobre la titularidad de los contenidos virtuales (es decir, algún proxy del marco legal de propiedad intelectual y las diversas situaciones normativa y contractuales) y, más precisamente, si se percibía un contraste entre la titularidad de los contenidos de la presencialidad y los de la educación online.

\section{Resultados de la encuesta: tiempo, conocimiento, remuneración y titularidad de los contenidos de la educación en línea}

Los resultados de la encuesta relevantes para este artículo se presentan alrededor de dos ejes. Por un lado, las representaciones de los docentes sobre las remuneraciones (como proxy del valor) de la producción de contenidos para educación online; por otro, las representaciones acerca de la titularidad de los contenidos. Ambas se relacionan, de distintos modos, con la ideología y la explotación. La asociación entre tiempo y valor, como señalamos, podría ser una asunción que favoreciera la explotación en situaciones en las que las ganancias dependen más de la reproducción de los conocimientos que de tiempos de trabajo. Las representaciones sobre la titularidad, por su parte, pueden habilitar u obstaculizar las relaciones de explotación. Si los docentes se representan la titularidad de los contenidos de la educación online como propia es más factible que esperen alguna remuneración por su uso reiterado. Por supuesto, ambos tipos de representación están vinculados: entre quienes asocian el valor con el tiempo de trabajo parece esperable una mayor expectativa de titularidad sobre la presencialidad (en la que el tiempo de trabajo es importante) que respecto de la reproducción de contenidos online (que se autonomizan del tiempo de su producción). 


\subsection{Representaciones sobre remuneraciones}

La tabla 1 muestra, sintéticamente, el resultado más importante de esta encuesta a los fines del presente trabajo: para los docentes el tiempo es una variable mucho más importante que el conocimiento a la hora de definir la remuneración esperada para un contenido digital. Pese a la cautela que la escasa cantidad de respuestas impone, se observa que el resultado es consistente tanto para los encuestados en el Reino Unido como en Argentina.

Tabla $\mathbf{n}^{\mathbf{0}}$ 1. Variable principal tenida en cuenta para determinar la remuneración esperada en el caso hipotético de preparar un curso online según país de trabajo de encuestados

(síntesis)

Reino Unido Argentina

\begin{tabular}{l|c|c|c|c|c|c}
\hline & $\mathrm{n}$ & $\%$ & $\mathrm{n}$ & $\%$ & $\mathrm{n}$ & $\%$ \\
\hline Tiempo & 67 & $73 \%$ & 24 & $69 \%$ & 91 & $71 \%$ \\
\hline $\begin{array}{l}\text { Conocimient } \\
\text { o }\end{array}$ & 25 & $27 \%$ & 11 & $31 \%$ & 36 & $29 \%$ \\
\hline Total & 92 & $100 \%$ & 35 & $100 \%$ & 127 & $100 \%$ \\
\hline
\end{tabular}

Fuente: Elaboración propia.

Total
Sin embargo, puede resultar interesante precisar las categorías de respuesta utilizadas y presentar resultados desagregados. Adicionalmente, es relevante añadir otra variable, dado que las representaciones sobre las remuneraciones podrían ser diferentes entre quienes efectivamente han tenido la experiencia de preparar cursos online y quienes no lo han hecho.

De este modo, en el gráfico 1 podemos encontrar las frecuencias y porcentajes de cada categoría de respuesta desagregada en función del país donde se ejerce la actividad y la existencia de experiencia de creación de contenidos para educación online. 
Gráfico $\mathbf{n}^{\mathbf{0}}$ 1. Variable principal tenida en cuenta para determinar la remuneración esperada en el caso hipotético de preparar un curso online, según país de trabajo de encuestados

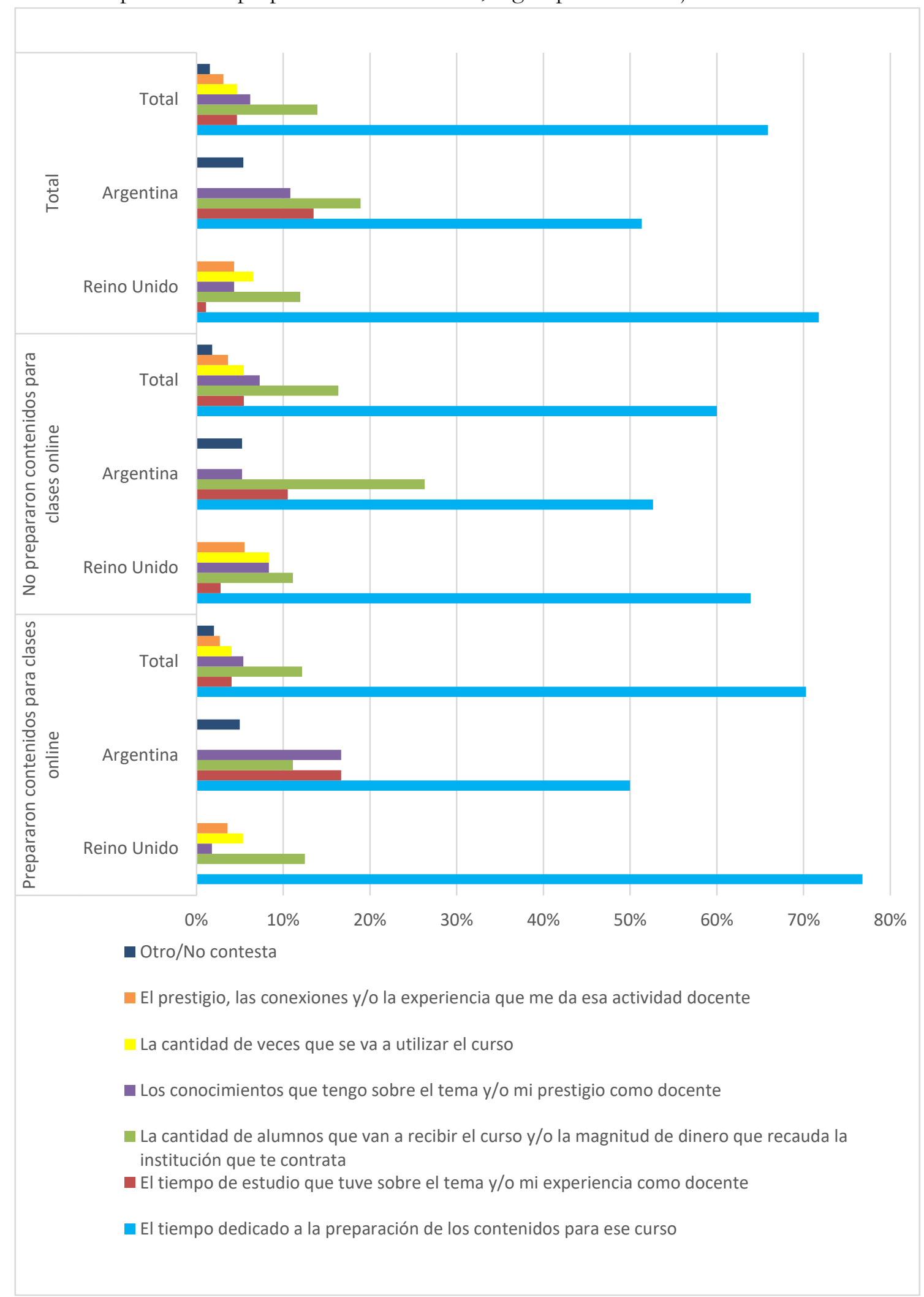

Fuente: Elaboración propia 
El gráfico 1 muestra que la opción más elegida en ambas encuestas es "el tiempo dedicado a la preparación de las clases" (promedio 66\%). En términos de diferencias entre países, si bien los encuestados del Reino Unido tendieron a elegir esta opción más que los argentinos ( $72 \%$ y 51\%, respectivamente), la otra categoría relativa al tiempo compensa parcialmente esa diferencia ( $0 \% \mathrm{y}$ $14 \%$ dedicados al tiempo de estudio y/o experiencia docente, respectivamente).

En cuanto a las categorías vinculadas al conocimiento, la opción explícitamente relacionada con los ingresos y el número de estudiantes fue la más seleccionada en ambos idiomas, un poco más elegida por los encuestados en español que por los de la versión en inglés $(19 \%$ y $12 \%$, respectivamente). El conocimiento del tema específico y el prestigio o reputación académica representan el $6 \%$ de las respuestas, siendo nuevamente mayor entre los encuestados en español $(11 \%$ vs $4 \%)$.

Tanto el incremento en el prestigio y la obtención de un cúmulo de atención como la cantidad de veces que el curso va a ser ofrecido concitaron porcentajes bajos en el Reino Unido (4\% y 6\%) y nulos en la Argentina.

Así, estos resultados son consistentes con nuestro argumento: la opción más seleccionada entre el tiempo es la que favorece la explotación a través de la reproducción. Por otro lado, la opción de conocimiento que potencialmente podría representar una mayor conciencia sobre la explotación a través de la reproducción solo representó un 5\% del total de encuestados.

En cuanto a la experiencia previa, más de la mitad de los encuestados habían preparado contenidos de cursos online (62\% en la encuesta en inglés y $49 \%$ en español). Si bien se trata de valores absolutos muy pequeños, es observa que no hay grandes discrepancias entre quienes han tenido experiencias y quienes no: el tiempo dedicado a la preparación de los cursos es la opción más elegida y las relativas al conocimiento se mantienen en valores muy modestos.

A su vez, aunque no se incluye en el gráfico por razones de espacio, si se consideran las áreas disciplinares en las que ejercen la docencia los encuestados tampoco se advierten cambios en las tendencias descriptas: aproximadamente el $70 \%$ tanto de quienes se ubican en las ciencias sociales (de las que por sus reflexiones sobre estos temas quizás cabía esperar representaciones diferenciales) como de quienes lo hacen en el resto de las disciplinas considera al tiempo como la principal variable relacionada con el valor de la remuneración esperada.

Finalmente, se preguntó por las razones para priorizar las categorías seleccionadas como fuente última de la remuneración esperada por la preparación de materiales para la educación online. 
Tabla $\mathbf{n}^{\circ}$ 2. Razones para priorizar variables que explican la remuneración esperada, según país de trabajo de los encuestados

\begin{tabular}{|c|c|c|c|c|c|c|}
\hline & \multicolumn{2}{|c|}{$\begin{array}{l}\text { Reino } \\
\text { Unido }\end{array}$} & \multicolumn{2}{|c|}{ Argentina } & \multicolumn{2}{|c|}{ Total } \\
\hline & $\mathrm{n}$ & $\%$ & $\mathrm{n}$ & $\%$ & $\mathrm{n}$ & $\%$ \\
\hline $\begin{array}{l}\text { Porque el pago se relaciona } \\
\text { con el tiempo de trabajo } \\
\text { presente y/o pasado. }\end{array}$ & 59 & $64 \%$ & 18 & $49 \%$ & 77 & $60 \%$ \\
\hline Otros & 19 & $21 \%$ & 5 & $13 \%$ & 24 & $19 \%$ \\
\hline $\begin{array}{l}\text { Porque el valor del curso } \\
\text { depende } \\
\text { conocimientos más que del } \\
\text { tiempo que le dedique }\end{array}$ & 5 & $5 \%$ & 10 & $27 \%$ & 15 & $11 \%$ \\
\hline $\begin{array}{l}\text { Porque desde esa institución } \\
\text { van a usar el curso } \\
\text { reiteradamente. }\end{array}$ & 9 & $10 \%$ & 3 & $8 \%$ & 12 & $9 \%$ \\
\hline $\begin{array}{l}\text { Porque es lo que consideran } \\
\text { otros/as colegas y/o el } \\
\text { sindicato }\end{array}$ & 0 & $0 \%$ & 1 & $3 \%$ & 1 & $1 \%$ \\
\hline Total & 92 & $100 \%$ & 37 & $100 \%$ & 129 & $\begin{array}{l}100 \\
\%\end{array}$ \\
\hline
\end{tabular}

Fuente: Elaboración propia

De acuerdo a la tabla el tiempo sigue siendo por mucho la opción más elegida en ambos idiomas, y el número sólo se reduce en un 11\% respecto de la tabla 1. Por su parte, el conocimiento se mantiene en valores bajos, aunque con gran dispersión en las encuestas en español e inglés. Los bajos valores absolutos impiden, sin embargo, avanzar en conclusiones sobre esta diferencia.

No obstante, la información nueva que aporta esta tabla refiere al 19\% de la opción "otros". Esto proviene de 13 encuestados que habían respondido "tiempo dedicado a la preparación...", 10 "Número de estudiantes..." у 2 "Aumento de su prestigio personal...". Con todo, las respuestas específicas en el espacio asignado confirman las opciones elegidas en las preguntas previas, es decir, no modifican la preeminencia del tiempo por sobre el conocimiento ni los pesos relativos de las distintas categorías.

Notablemente, la influencia de colegas o sindicatos no parece ser relevante en este tópico para los encuestados.

\subsection{Representaciones sobre titularidad de los contenidos}

Esta sección aborda las representaciones respecto de quién es el titular (en el sentido de propietario, no en el de responsable a cargo de un curso) de los contenidos desarrollados para la educación. Particularmente, interesa contrastar las representaciones acerca de los contenidos de la educación en línea con los de la educación presencial. 
A diferencia de la subsección anterior, aquí hay antecedentes que conviene mencionar brevemente. En un estudio realizado por Aaron y Roche (2015) entre profesores de un colegio comunitario, los investigadores incluyeron una pregunta específica para los encuestados que no pensaban que todo el material producido por académicos debería ser de su titularidad. La pregunta se refería a la titularidad de diversas clases de obras de autoría. Aunque el número de encuestados fue extremadamente bajo para esta pregunta en particular $(\mathrm{n}=19)$, los resultados resultaron ser sugerentes: al 79\% (15) respondió que los artículos publicados en revistas revisadas por pares podrían ser propiedad de académicos, el 37\% (7) lo hizo con respecto al aula tradicional contenidos y solo el 16\% (3) cuando se trata de clases online. ¿Por qué esta última cifra es mucho más baja que la anterior? Desafortunadamente, el estudio no indagó en esta cuestión. Sin embargo, podría argumentarse que dado que los materiales son bastante similares desde una perspectiva legal, las diferencias son hasta cierto punto explicables por alguna clase de discurso ideológico. Mientras que ese estudio preguntó quién debería ser el propietario (una pregunta prescriptiva), otro indagó sobre las representaciones acerca de quién es el titular (de manera más cercana a nuestro interés) y encontró resultados contrastantes.

Así, cuando se les preguntó si poseían derechos de propiedad intelectual sobre el contenido que habían producido para sus propios MOOC, el 73,3\% de los 100 encuestados respondió "Sí", el 11,9 respondió "No" y el 14,9\% eligió " No lo sé "(Kolowich, 2013). Es importante considerar que la media de estudiantes de esos cursos fue de 33000; así, podría haber alguna relación entre la masividad y la asociación con el valor del conocimiento y la expectativa de titularidad.

En cuanto a nuestras encuestas, planteamos dos preguntas muy generales, para captar algunas representaciones sobre la titularidad y, en particular, para comparar las representaciones sobre contenidos en línea y presenciales. Como se mencionó en la sección metodológica, los resultados deben tomarse con cautela.

Tabla $\mathbf{n}^{\mathbf{0}}$ 3. Representaciones acerca de la titularidad de las clases presenciales y los cursos en línea

\section{Reino Unido}

Argentina

Total

\begin{tabular}{|c|c|c|c|c|c|c|c|c|c|c|c|c|}
\hline & \multicolumn{2}{|c|}{ Presenciales } & \multicolumn{2}{|c|}{ A distancia } & \multicolumn{2}{|c|}{ Presenciales } & \multicolumn{2}{|c|}{ A distancia } & \multicolumn{2}{|c|}{ Presenciales } & \multicolumn{2}{|c|}{ A distancia } \\
\hline & $\mathrm{n}$ & $\%$ & $\mathrm{n}$ & $\%$ & $\mathrm{n}$ & $\%$ & $\mathrm{n}$ & $\%$ & $\mathrm{n}$ & $\%$ & $\mathrm{n}$ & $\%$ \\
\hline $\begin{array}{l}\text { La } \\
\text { institución } \\
\text { que } \\
\text { contrató al } \\
\text { docente }\end{array}$ & 29 & $32 \%$ & 51 & $55 \%$ & 4 & $11 \%$ & 18 & $49 \%$ & 33 & $26 \%$ & 69 & $53 \%$ \\
\hline El docente & 39 & $42 \%$ & 28 & $31 \%$ & 15 & $41 \%$ & 7 & $19 \%$ & 54 & $42 \%$ & 35 & $27 \%$ \\
\hline Nadie & 22 & $24 \%$ & 12 & $13 \%$ & 18 & $49 \%$ & 12 & $32 \%$ & 40 & $31 \%$ & 24 & $19 \%$ \\
\hline $\begin{array}{l}\text { Los } \\
\text { estudiantes }\end{array}$ & 2 & $2 \%$ & 1 & $1 \%$ & 0 & $0 \%$ & 0 & $0 \%$ & 2 & $2 \%$ & 1 & $1 \%$ \\
\hline Total & 92 & $100 \%$ & 92 & $100 \%$ & 37 & $100 \%$ & 37 & $100 \%$ & 129 & $100 \%$ & 129 & $100 \%$ \\
\hline
\end{tabular}

Fuente: Elaboración propia. 
Con respecto a las clases presenciales, la opción más seleccionada fue "el profesor" (42\%), mostrando resultados similares en ambos idiomas. "Nadie" ocupó el segundo lugar (31\%). Sin embargo, los resultados de ambas encuestas fueron bastante diferentes. Mientras que en la encuesta en inglés esta opción fue elegida por el $24 \%$ de los encuestados, el $49 \%$ de los encuestados en español la escogió, ocupando el primer lugar en este idioma. Una diferencia similar pero en la dirección opuesta se encuentra respecto de "la institución": la proporción de encuestados del Reino Unido que la eligió es del 32\% mientras que los encuestados argentinos solo representan el 11\%.

En cuanto a los cursos en línea, el 53\% de los encuestados respondió a la pregunta abstracta sobre la titularidad eligiendo "la institución". Las cifras son bastante similares para las encuestas en inglés (55\%) y en español (48\%). Las opciones en segundo y tercer lugar fueron "el docente" y "nadie", respectivamente. Sin embargo, aquí hubo importantes diferencias entre las encuestas. Mientras que los profesores fueron un poco más seleccionados en la encuesta en inglés que en la española (30\% frente a 19\%), con "nadie" fue al revés (13\% frente a 32\%). Esto debería estar enmarcado por el hecho de que los encuestados españoles tendían a elegir la opción "nadie" mucho más que los encuestados en inglés con respecto a las clases presenciales también.

En suma, el resultado más importante que muestra este cuadro es que mientras para los contenidos asociados a la presencialidad la titularidad corresponde a la institución para el $26 \%$ de los encuestados, esta cifra se eleva al 53\% cuando se trata de contenidos en línea. El contraste es notable porque es precisamente mediante la titularidad de contenidos en línea que la institución puede multiplicar sus ingresos y sugiere una base firme para la explotación por reproducción.

Para captar de manera específica la existencia o no de contraste entre la titularidad esperada para los materiales de enseñanza en línea y los presenciales, agrupamos los resultados de combinar ambas respuestas por parejas.

$\operatorname{Tabla~}^{\circ}{ }^{\circ}$. Respuestas coincidentes o diferentes respecto de la titularidad de las clases presenciales y los cursos en línea

\begin{tabular}{c|c|c|c|c|c|c} 
& \multicolumn{2}{c}{ Reino Unido } & \multicolumn{2}{c}{ Argentina } & \multicolumn{2}{c}{ Total } \\
\hline & $\mathrm{n}$ & $\%$ & $\mathrm{n}$ & $\%$ & $\mathrm{n}$ & $\%$ \\
\hline $\begin{array}{c}\text { Misma } \\
\text { respuesta }\end{array}$ & 64 & $70 \%$ & 23 & $62 \%$ & 87 & $67 \%$ \\
\hline $\begin{array}{c}\text { Respuestas } \\
\text { diferentes }\end{array}$ & 28 & $30 \%$ & 14 & $38 \%$ & 42 & $33 \%$ \\
\hline Total & 92 & $100 \%$ & 37 & $100 \%$ & 129 & $100 \%$ \\
\hline
\end{tabular}

Fuente: Elaboración propia.

El resultado principal es que una gran mayoría de los encuestados en ambas encuestas $(67 \%)$ tendió a elegir las mismas opciones con respecto a las clases presenciales y en línea. Esto sugiere que para una parte importante de los académicos, la titularidad de un servicio (clases presenciales) y la de bienes informacionales (cursos en línea) son similares, es decir, que el soporte material sobre el cual existe el conocimiento no está relacionado con las regulaciones. Sin embargo, esto es usualmente incorrecto. Sobre los contenidos codificados y objetivados en un 
soporte digital se obtienen derechos de autor, mientras que sobre los servicios no. Este posible error invita a recorrer un curso de acción político para los interesados en luchar contra la explotación por reproducción: brindar información legal y discutir con los académicos las consecuencias legales, económicas e incluso ontológicas de objetivar sus clases como bienes informacionales.

\section{Conclusiones}

La educación en línea con fines de lucro avanza a un ritmo desparejo pero inexorable. Su presencia en la educación superior ya era importante antes de la pandemia de COVID 19, pero durante ella se ha expandido notablemente en los más diversos ámbitos nacionales. Más allá de las virtudes y limitaciones de estas modalidades en términos pedagógicos aquí nos interesa otro aspecto mucho menos discutido. En efecto, en este artículo hemos intentado acercarnos a la situación de los productores de contenidos para diferentes modalidades de educación online desde la perspectiva de la explotación capitalista, y particularmente de la explotación por reproducción a través de la reproducción y las normativas e ideología que la enmarcan.

A la hora de las conclusiones es importante señalar que la explotación por reproducción en el ámbito de la educación en línea es muy parecida a la que ocurre en muchos otros ámbitos. En efecto, la historia del capitalismo está plagada de situaciones en las que algunos actores, en busca de lucro, copian conocimientos desarrollados o acarreados por otros actores, sin brindar una compensación acorde con el valor que obtienen. Sin embargo, la explotación por reproducción en el capitalismo informacional adquiere algunas características originales. Esta etapa se define en parte por la masiva expansión de diferentes derechos de propiedad intelectual en las más diversas variables (Zukerfeld, 2017c). No obstante y notablemente, la explotación por reproducción viola o elude lo que podrían parecer derechos de propiedad intelectual pertenecientes a los autores de las obras. En otras palabras, en un contexto de expansión del derecho de autor, la explotación por reproducción prospera en base en negar ese derecho a algunas obras y titulares. Para ello, la explotación por reproducción descansa en herramientas ideológicas que se diferencian y, en cierta medida, son contradictorias con las que actúan en defensa de la propiedad. Esta aparente contradicción puede entenderse mejor si se considera que el capitalismo informacional, en realidad, presenta al menos dos fases. La primera, la fase de las redes, que va desde los años 1970 hasta mediados de los 2000, está marcada por esta expansión de la modalidad privativa y excluyente de la propiedad intelectual y los discursos del individualismo y los incentivos. La segunda, fase de las plataformas y que se extiende desde entonces hasta el presente, se corresponde con modelos de negocios capitalistas basados en la apertura, las comunidades, el estímulo a las prácticas de compartir y, asociado a ello, la explotación por reproducción. Por ejemplo, esto ocurre con los contenidos subidos a YouTube, con algunos modelos de negocio basados en la apropiación no remunerada del Software Libre, y muchos otros casos en los que un discurso a favor de la libertad encubre la negativa a pagar a los productores una compensación acorde a las magnitudes de valor que generan.

En el caso de los productores de contenidos educativos digitales el punto crucial es que mediante diversos dispositivos legales e ideológicos quienes producen las clases, que en principio son los titulares desde el momento en que la obra se fija en un medio tangible, terminan perdiendo sus derechos en lo que respecta a la reproducción de dichas obras. 
No obstante, las herramientas ideológicas que operan aquí no son exactamente las mismas que en los casos ya mencionados (YouTube, software libre, etc.): aquí queremos llamar la atención sobre el papel que en este sentido juega la asociación que muchos trabajadores de la educación hacen entre el origen del valor (y por ende de la remuneración esperada) y el tiempo de preparación de la clase. Esta asociación es útil para medir (y luchar contra) la denominada explotación por la enajenación (la explotación tradicional identificada por Marx en el capitalismo industrial). Es la modalidad de explotación la que se da en las clases presenciales, provistas como un servicio que debe demandarse y remunerarse cada vez que se provee, como conocimiento vivo. Pero la explotación por enajenación y su supuesto de relación entre tiempo y valor se vuelven insuficientes para dar cuenta de que los contenidos se repiten como conocimiento muerto una y otra vez, sin dependencia necesaria de aquellos trabajadores que originalmente los produjeron.

Así, aunque nuestro trabajo de campo ha sido sumamente limitado nos permite plantear una idea que otras investigaciones habrán de ratificar o rectificar: la amplia asociación por parte de los docentes entre el remuneración (como proxy del valor) por la producción de contenidos digitales y el tiempo que se tarda en elaborar dichos contenidos, representa una base ideológica significativa a partir del cual las instituciones con fines de lucro que producen estos cursos pueden avanzar en la explotación por reproducción de esos docentes.

Este es, entonces, el hallazgo más importante y controvertido del presente artículo. Asociar la remuneración con el tiempo de trabajo no solo es teóricamente incorrecto en este caso, sino que podría ser parte de la ideología dominante de la presente fase del capitalismo informacional que, como tal, ayuda a aceptar y naturalizar las relaciones de explotación.

¿Cómo debatir y combatir la explotación a través de la reproducción? El primer paso consiste, posiblemente, en difundir información relacionada con los derechos de propiedad intelectual, y en particular los derechos de autor. Si bien esta investigación no permite afirmaciones vehementes sobre el nivel de conocimiento que tienen los docentes sobre la normativa vigente, hay elementos que sugieren que su base en tales materias a menudo es insuficiente para defender sus intereses individuales y colectivos. Es decir, incluso dentro de la legalidad capitalista, el desconocimiento de la normativa sobre derechos de autor facilita la explotación por reproducción; asimismo, difundir modelos de cláusulas y contratos justos para la producción, reproducción y remuneración de contenidos online parece imperativa.

Por supuesto, los docentes de la educación superior están lejos de ser una excepción y esto también se aplica a otros casos de explotación mediante la reproducción. La difusión y reforma de las leyes de propiedad intelectual y los modos de lucha contra la apropiación capitalista del conocimiento son tareas políticas cruciales en el capitalismo informacional. Un paso más, dentro de los acotados límites de la sociedad capitalista consistiría en regular estas actividades con una legislación específica que regulara la reproducción no remunerada de conocimientos con fines de lucro.

El destino de la educación superior con fines de lucro depende de varias fuerzas. Quiero mencionar dos de ellas aquí. Una es la medida en que las empresas pueden extraer plusvalía de los docentes y otros productores de contenido. Tal medida está relacionada, a su vez, con el grado de organización política de los trabajadores docentes y la capacidad de sus representantes para obtener mejores remuneraciones. Esta cuestión no es independiente de la dinámica del empleo y los salarios. Así, aunque no se haya abordado en este trabajo, es evidente que la aceptación, naturalización e incluso entusiasmo de los docentes por involucrarse en relaciones de 
explotación por reproducción no está desvinculada con la precarización general del trabajo y la modestia de los salarios docentes. Esta precarización evidentemente ocurre en la Argentina (y América Latina) pero también en el Reino Unido (Europa y otros ámbitos centrales).

La otra fuerza alude al grado de circulación compartida (sharing) de los contenidos, es decir, la medida en la que se vuelven bienes comunes o cuasi públicos. Es sabido que las ganancias corporativas se ven amenazadas por el avance de los bienes comunes (informacionales) tanto como que los ecosistemas de los bienes comunes (informacionales) se ven amenazados por las actividades comerciales -que sesgan sus contenidos hacia fines lucrativos, los inundan de publicidad, etc.-. Así, este campo, como muchos otros, está conformado por la interacción dialéctica y las disputas abiertas entre las tendencias a la comunalización y la mercantilización.

¿Cómo pensar la relación entre la digitalización de los contenidos y el trabajo docente? ¿Deben los docentes, al fin y al cabo, rechazar la digitalización de los contenidos para preservar sus fuentes de trabajo? Al fin y al cabo, parece claro que la digitalización permite el acceso a la educación a estudiantes que de otro modo -en pandemia, pero no sólo en ella- no podrían acceder a ella. Parecería entonces que no se trata necesariamente de rechazar la digitalización de los contenidos sino, sobre todo, de contrarrestar su mercantilización y luchar por que formen parte de la riqueza colectiva de los bienes informacionales comunes.

Es fácil estar de acuerdo con la idea de los bienes comunes educativos. Sin embargo, es una tarea mucho más complicada considerar qué sucede con los docentes en un escenario en el que una parte sustantiva de los contenidos que producen se convierten en bienes informacionales comunes. Aquí no es posible explorar las múltiples propuestas políticas posibles (reciclaje en el trabajo de investigación, la idea de una renta básica universal, etc.), pero podemos señalar que, en cada caso, bajo el capitalismo, el eventual trade-off entre la inclusión social que genera la comunalización del conocimiento y la pérdida de salario que esto podría implicar para los trabajadores de la educación debe ser analizado con cuidado.

\section{Referencias}

Aaron, Lynn S., \& Roche, Catherine M (2015) Intellectual Property Rights of Faculty in the

Digital Age-Evolution or Dissolution in 21st Century Academia? Journal Of Educational Technology Systems, 43(3), 320-341. https:// doi.org/10.1177/0047239515570582

Anderson, T. (2008) The theory and practice of online learning. Athabasca University Press.

Borrego, Nali; Rodríguez, Humberto; Walle, Rogelio and Ponce, Jesus. 2008. Educación Superior

Virtual en América Latina: Perspectiva Tecnológica-Empresarial. Formación universitaria, 1(5), 3-14. https://dx.doi.org/10.4067/S0718-50062008000500002

Browne, T., Jenkins, M., and Walker, R. (2006). A longitudinal perspective regarding the use of

VLEs by higher education institutions in the United Kingdom. Interactive Learning Environments, 14(2), 177-192.

Castillo, L. M. (2020). Lo que la pandemia nos enseñó sobre la educación a distancia. Revista Latinoamericana de Estudios Educativos (México), 50, 343-352.

Chang, H.J. (2001) Intellectual property rights and economic development: Historical Lessons and Emerging Issues, Journal of human development, 2(2), 287-309.

Cooper, S. y Sahami, M. (2013) Reflections on Stanford's MOOCs. Communications of the $A C M, 56(2), 28-30$.

Coriat, B. (1985). El taller y el cronómetro. Ensayo sobre el taylorismo, el fordismo y la producción en masa. 
Siglo XXI.

Dolcemáscolo, A. (2014). Explotación Cognitiva en plataformas de video: El caso de YouTube. Hipertextos, 3(2), pp. 43-67.

Dolcemáscolo, A. y Yansen, G. (2017) Informational cognitive exploitation: concealed relationships behind prosumers' activity on the World Wide Web, Sociologia del lavoro, n. 145/2017. pp. 61-77. DOI: 10.3280/SL2017-145004. Recuperado de https://www.francoangeli.it/riviste/Scheda_Rivista.aspx?IDArticolo=58742\&Tipo=Articol $\mathrm{O} \% 20 \mathrm{PDF} \&$ idRivista $=83$

Drahos, P. y Braithwaite, J. (2002) Information Feudalism: Who owns the knowledge economy? The New Press.

Elster, J. (1981) “Roemer vs. Roemer”, Politics \& Society, 11:3.

Fisher, E. (2012) How Less Alienation Creates More Exploitation? Audience Labour on Social Network Sites, tripleC - Open Access Journal for a Global Sustainable Information Society ,10 (2), 171 183. https://doi.org/10.31269/triplec.v10i2.392

Fuchs, Ch. (2012) With or without Marx? With or without capitalism? A rejoinder to Adam Arvidsson and Eleanor Colleoni, tripleC - Open Access Journal for a Global Sustainable Information Society 10 (2), 633-645

Fuchs, Ch. (2015) Against Divisiveness: Digital Workers of the World Unite! A Rejoinder to César Bolaño and Eloy Vieira, Television \& New Media 16 (1), 62-71.

Fuchs, Ch. (2010). Labor in informational capitalism and on the Internet. The Information Society 26 (3), 179-196.

Fumagalli, A (2015) The concept of life subsumption of labour to capital: towards the life subsumption in bio-cognitive capitalism, forthcoming. En E. Fisher, C. Fuchs (eds.), Reconsidering value and labour in the digital age. Palgrave-McMillan.

Gagliardi, V. (2020). Desafíos educativos en tiempos de pandemia. Questión.

Garegnani, P.A. (1979) La realidad de la explotación, Debate sobre la teoría marxista del valor. Cuadernos de Pasado y Presente, 82.

Geteducated.com. (2016) Teaching online courses. Get.educated.com. https://www.geteducated.com/teaching-online-courses/253-online-teaching-opportunities

Harasim, L. (1996). Online education. Computer networking and scholarly communication in the twentyfirst-century university, 203-214.

Hilferding, R. ([1910] 1981). Finance Capital. Routledge \& Kegan Paul.

Hodgson, G. (1988) Una teoría de la explotación sin la teoría del valor trabajo. Revista Economía Teoría y Politica. No 12, 141-153.

Hoxby, C. (2014) The Economics of Online Postsecondary Education: MOOCs, Nonselective Education, and Highly Selective Education, American Economic Review, 104(5), 528-33.

Izumi, T., Sukhwani, V., Surjan, A., \& Shaw, R. (2020). Managing and responding to pandemics in higher educational institutions: initial learning from COVID-19. International Journal of Disaster Resilience in the Built Environment.

Johns, A. (2010). Piracy. The Intellectual Property Wars from Gutenberg to Gates. University of Chicago Press.

Kolowich, S. (2013) "The Professors Who Make the MOOCs". Chronicle of Higher Education. Retrieved 26 March 2013. 
Kreimer, P. y Zukerfeld, M. (2014) La explotación cognitiva: Tensiones emergentes en la producción y uso social de conocimientos científicos tradicionales, informacionales y laborales. En Kreimer, Vessuri, Velho y Arellano Perspectivas latinoamericanas en el estudio social de la ciencia, la tecnología y el conocimiento. Siglo XXI.

Lazzarato, M. y Negri. A. (2001) Trabajo inmaterial. Formas de vida y producción de subjetividad. DP\&A Editora.

Lee, Doo Yung, y Lehto, Mark. R. (2013) User acceptance of YouTube for procedural learning: An extension of the Technology Acceptance Model. Computers \& Education, 61, 193-208.

Liaudat, S. (2021) Stevia. Conocimiento, propiedad intelectual y acumulación de capital. Prometeo.

Marotias, A. (2020). La educación remota de emergencia y los peligros de imitar lo presencial. Hipertextos, 8 .

Marx, K. ([1867] 1990) Capital: A Critique of Political Economy, vol. 1. Penguin.

May, Ch. y Sell, S. K. (2006) Intellectual property rights: a critical history. Boulder, Colorado: Lynne Riener Publishers.

Mishra, L., Gupta, T., \& Shree, A. (2020). Online teaching-learning in higher education during lockdown period of COVID-19 pandemic. International Journal of Educational Research Open, 1, 100012.

Moulier-Boutang, Y. (2011) Cognitive Capitalism. Polity Press.

Rabosto, A. (2014). Apuntes para una crítica de los estudios de la web. Hipertextos, 2(1), 30-63.

Roemer, J. E. (1985) Should Marxists be Interested in Exploitation? Philosophy and Public Affairs, 14 (1), 30-65.

Salmon, G. 2013. E-tivities: The key to active online learning. Routledge.

Santamans, J.M. 2014. El mercado Global de e-learning, Online Business School. Available at: https://gcu.universia.net/net/files/2014/6/21/investigacion-obs-el-mercado-global-del-elearning-2014.pdf

Selwyn, N. (2007) Web 2.0 applications as alternative environments for informal learning-a critical review. En Paper for CERI-KERIS International Expert Meeting on ICT and Educational Performance (pp. 16-17).

Simon, H. A. (1996) The Sciences of the Artificial. The MIT Press.

Smythe, D.W. (1977) Communications: blindspot of western Marxism, Canadian Journal of Political and Social Theory, 1(3), 1-27

Sweezy, P.M. (1942) The Theory of Capitalist Development. Monthly Review Press.

Torres, Patricia L. Y Rama, Claudio (eds) (2010). La educación superior a distancia en América Latina y el Caribe. Editora Unlsul.

U.S. Department of Education, National Center for Education Statistics. (2016) Digest of Education Statistics, 2014 (NCES 2016-006), Table 311.15. Available at: https://nces.ed.gov/fastfacts/display.asp?id=80

Vercellone, C. (2011). Capitalismo cognitivo: renta, saber y valor en la época posfordista. Prometeo.

Weller, M.(2002) Delivering learning on the Net: The why, what \& how of online education. Psychology Press.

Wright, E.O. ([1985] 1997) Classes. Verso. 
Yansen, G. (2015) Explotación cognitiva informacional: un analisis de una plataforma web de contenido audiovisual. Tesis de Maestría. FLACSO. Sede Académica Argentina, Buenos Aires. http://hdl.handle.net/10469/7864

Zhou, L., Wu, S., Zhou, M., \& Li, F. (2020). 'School's Out, But Class' On', The Largest Online Education in the World Today: Taking China's Practical Exploration During The COVID-19 Epidemic Prevention and Control As an Example. Best Evid Chin Edu, 4(2), 501-519.

Zukerfeld, M. (2017a) Exploitation, Regulation and Ideology in Online Education: Towards a Theory of Exploitation through Reproduction in Informational Capitalism. Westminster Advanced Studies, 9. London: Westminster Institute for Advanced Studies

Zukerfeld, M. (2017b). Knowledge in the Age of Digital Capitalism: An Introduction to Cognitive Materialism. University of Westminster Press.

Zukerfeld, M. (2017c). The tale of the snake and the elephant: Intellectual property expansion under informational capitalism. The Information Society, 33 (5), 243-260.

Zukerfeld, Mariano (2014) Inclusive Appropriation and the Double Freedom of Knowledge: On the Capitalist exploitation of non-for profit software, contents and data producers. En Special Issue Free and Unpaid Work: Gratuity, Collaborative Activity And Precariousness, Sociología del Lavoro, (133), 144-158

Zukerfeld, M. (2010) Capitalismo y Conocimiento. Materialismo Cognitivo, Propiedad Intelectual y Capitalismo Informacional. Tesis de doctorado en Flacso Argentina. Disponible en capitalismoyconocimiento.wordpress.com. 